\title{
SYSTEMATIZATION OF THEORETICAL-METHODOLOGICAL APPROACHES TO NEW INDUSTRIAL POLICIES
}

\section{EKA LEKASHVILI}

Academic Doctor of Economics, Associate Professor

Ivane Javakhishvili Tbilisi State University, Georgia

eka.lekashvili@tsu.ge

Abstract. Economic policy is a constant resistance between economic and theoretical perspectives and political expediency. Industrial policy arise more on the basis of a normative approach as it aims to improve the economic situation of the country. The new industrial policy means the formation of firms by the state, their aggregation, support of innovations and competitive advantages in frame of open economy.

The goal of the research is to study the theoretical and methodological approaches of new industrial policies in order to form a theoretical framework for assessing practical industrial policy.

In the concepts of new industrial policy, we consider the endogenous theory of growth, based on the level of knowledge and research activity in the country; National Competitive Advantage Theory; National Innovation Systems Concept, which is based on the impact of the national economic-political situation, trade policy, education sector and institutions on innovation. Furthermore, we will analyze the results of advanced research on new industrial policy issues.

KEYWORDS: NEW INDUSTRIAL POLICY THEORETICAL APPROACHES

For citation: Lekashvili, E. (2020). Systematization of theoretical-methodological approaches to new industrial policies. Globalization and Business, №9, pp. 27-34. (In Georgian) https://doi.org/10.35945/gb.2020.09.003 


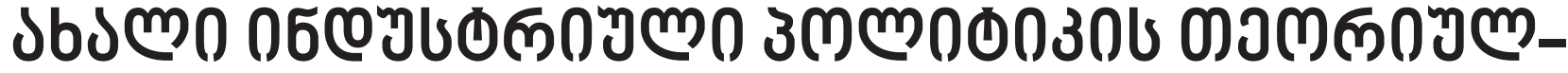

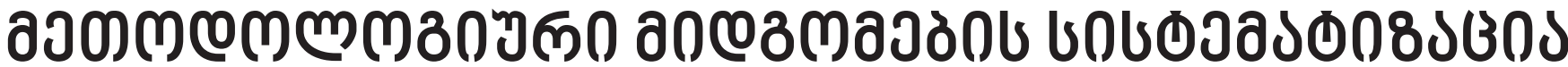

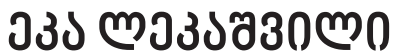

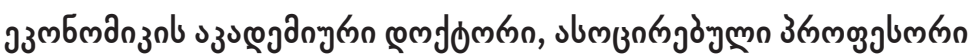

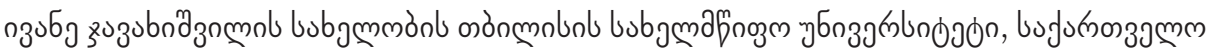

eka.lekashvili@tsu.ge

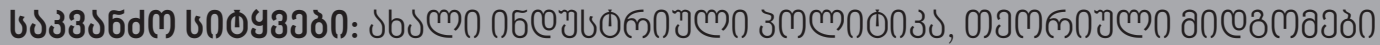

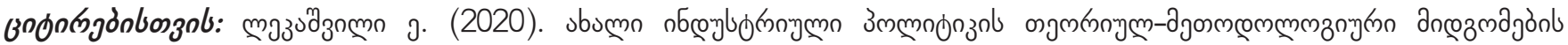

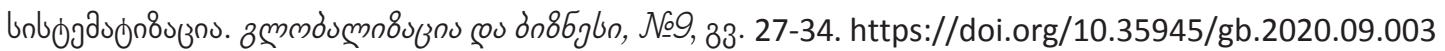

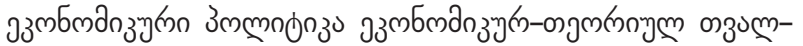

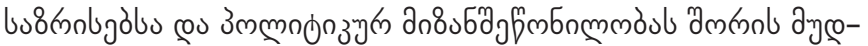

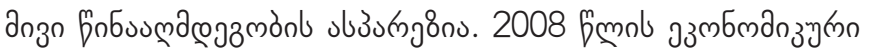

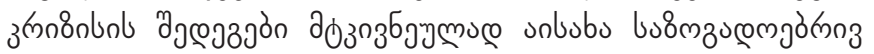

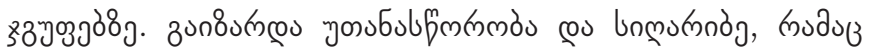

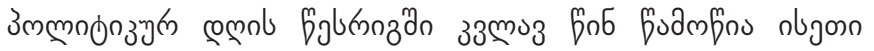

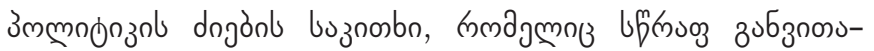

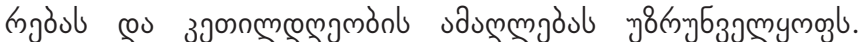

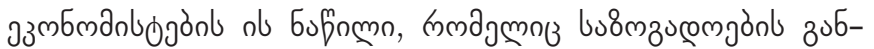

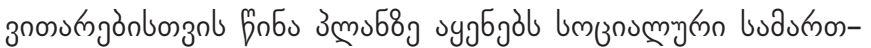

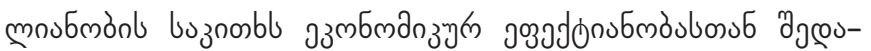

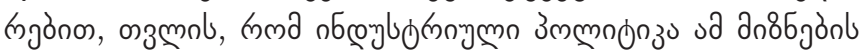

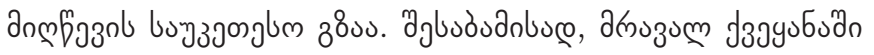

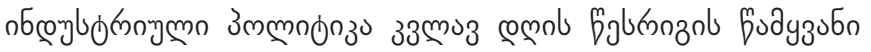

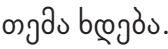

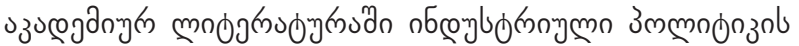

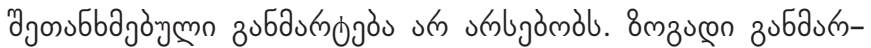

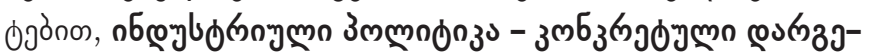

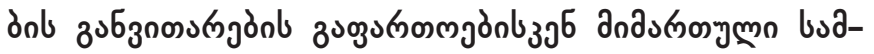

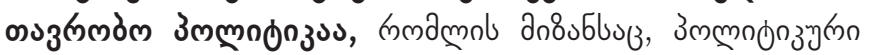

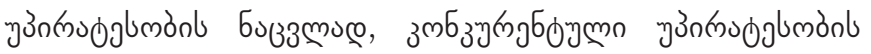

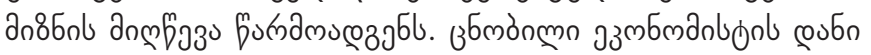

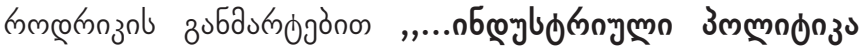

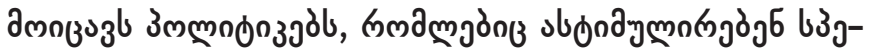

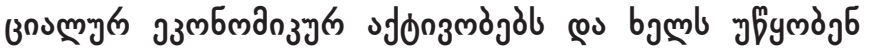

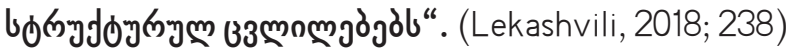

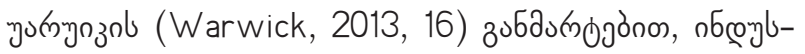

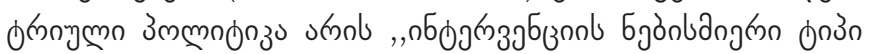

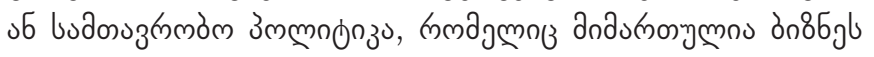

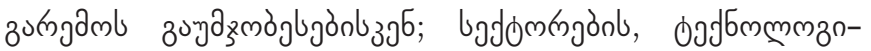

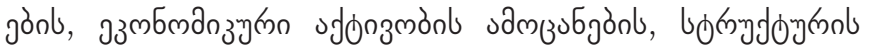

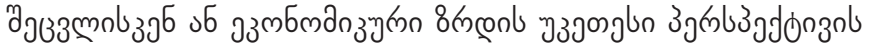

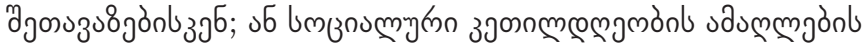

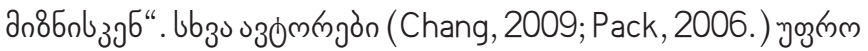

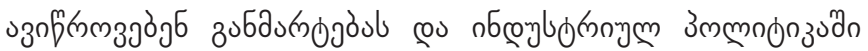

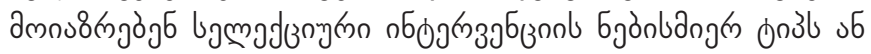

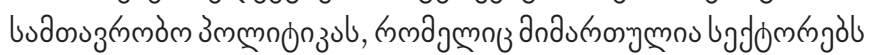

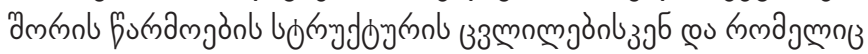

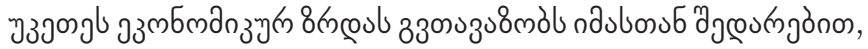

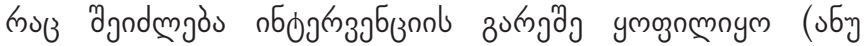

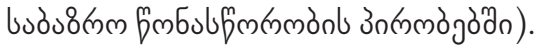

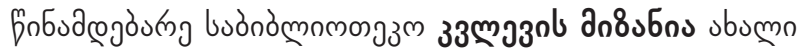
nбеу

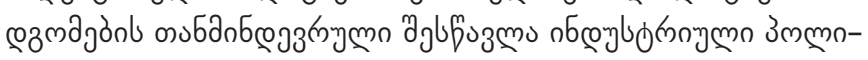

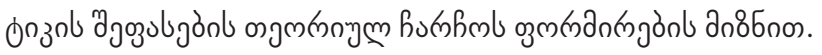

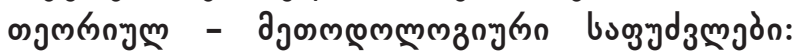

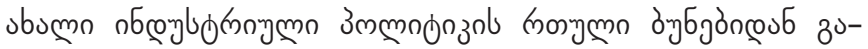

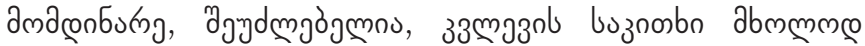

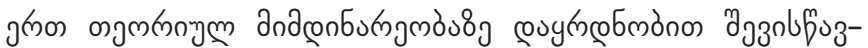

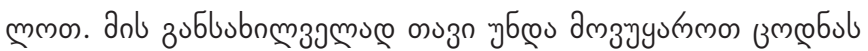

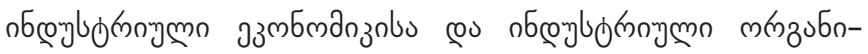

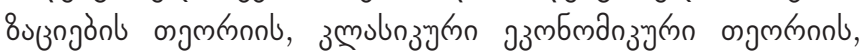
s зb

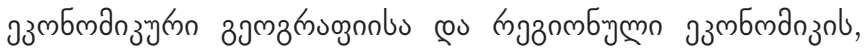

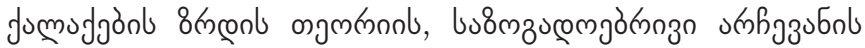

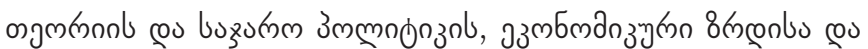

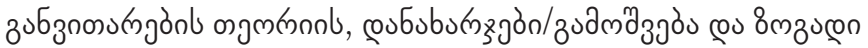

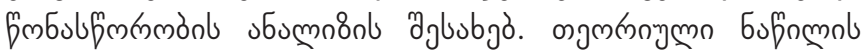

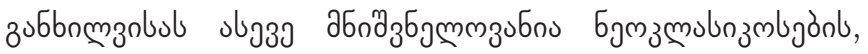

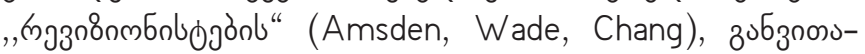

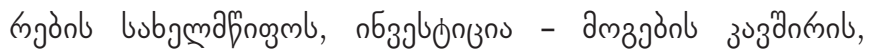

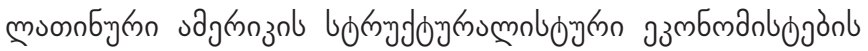

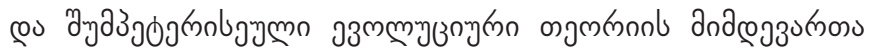
๓зumbusmolgàn. 


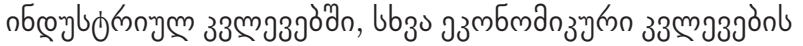

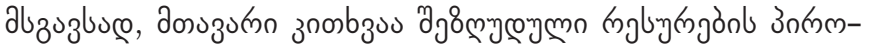

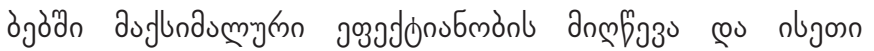

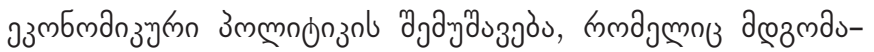

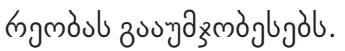

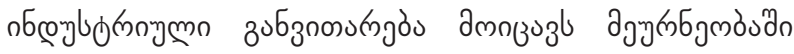

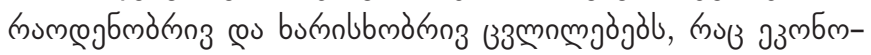

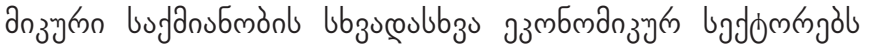

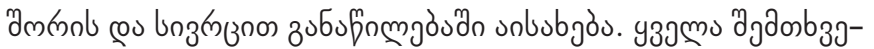

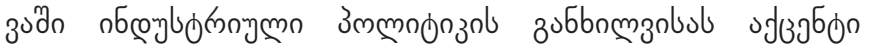

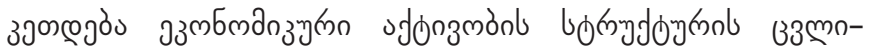
mgos

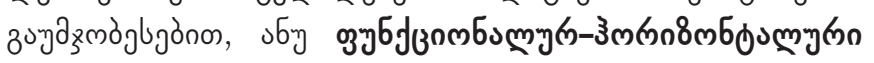

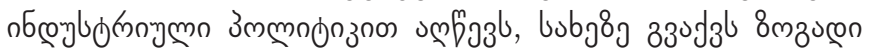

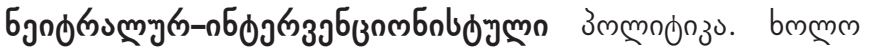

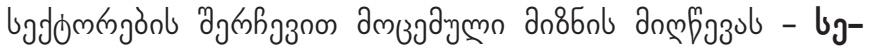

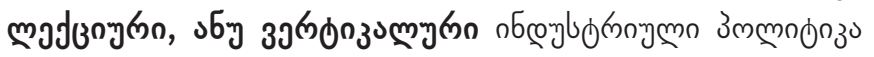

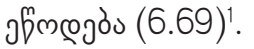

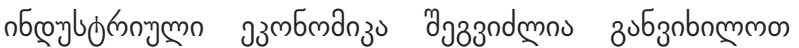

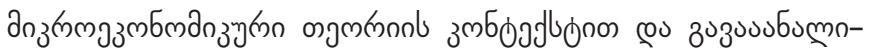

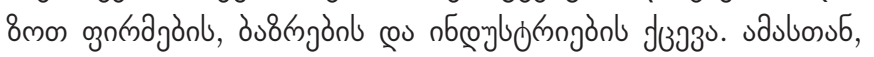

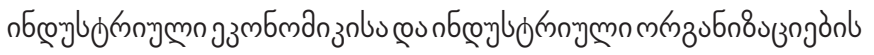

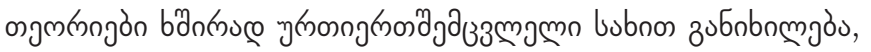

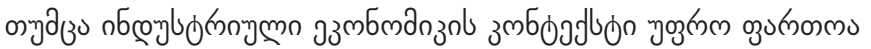

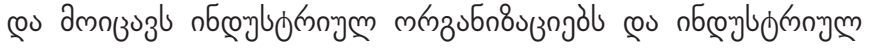

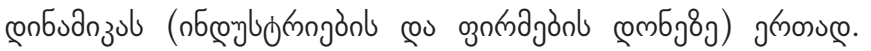

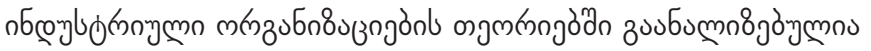
bringmymoुnmn змб

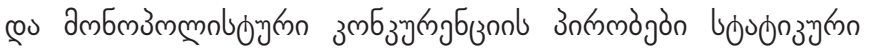

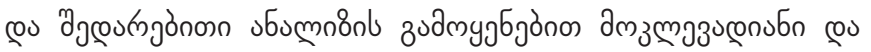

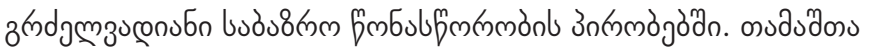

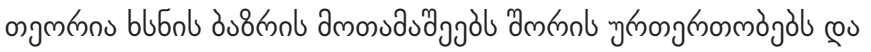

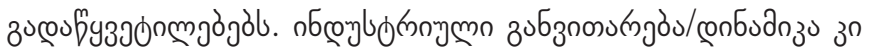

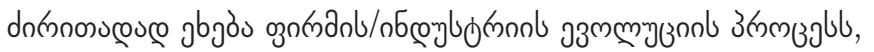

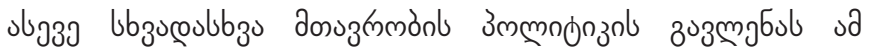

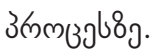

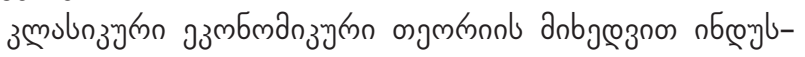

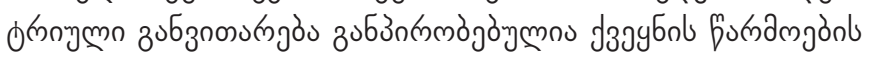

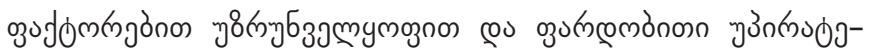

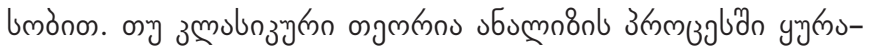

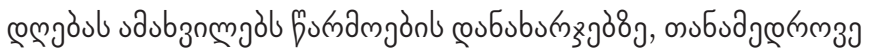

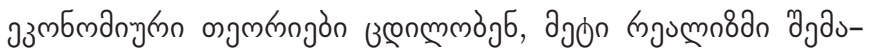

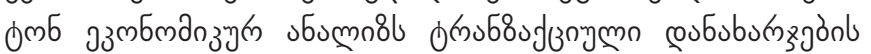
zuonzumn uf

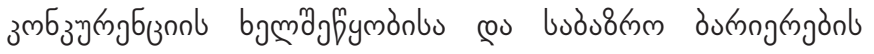

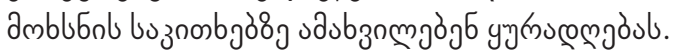

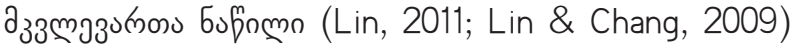

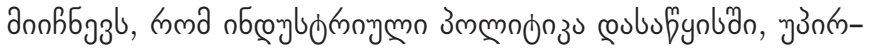

${ }^{1}$ Industrial policy: a theoretical and practical framework to analyses and apply industrial policy. Module 2. 67-132. https://vi.unctad.org/stind/m2.pdf

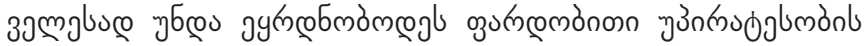

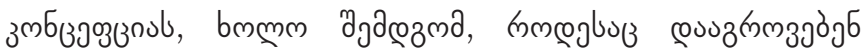

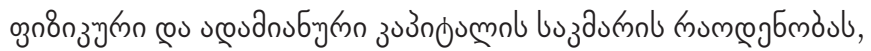

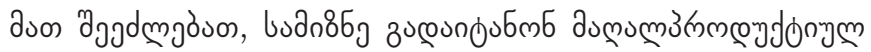

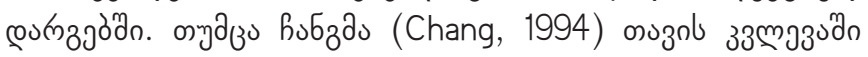

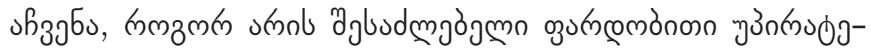

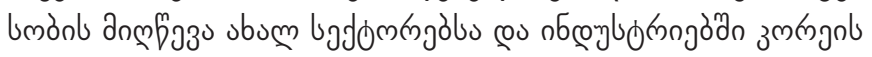

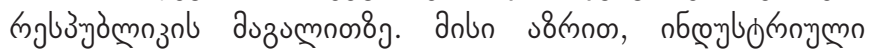

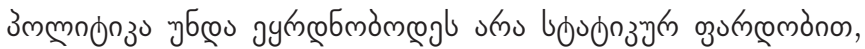

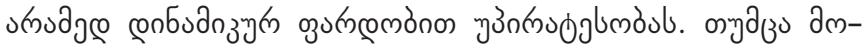

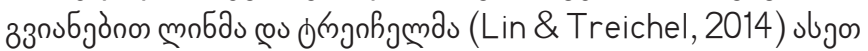

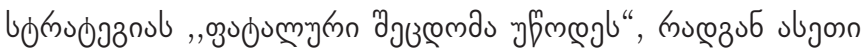

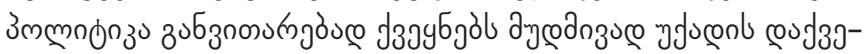

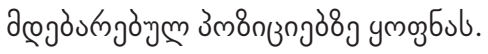

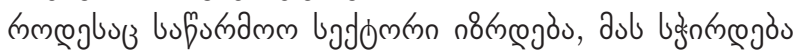

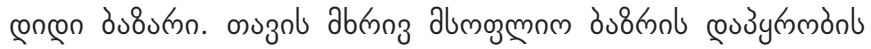

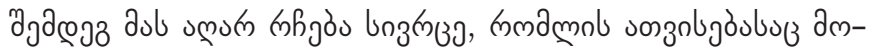

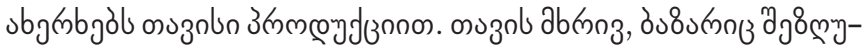

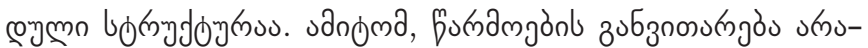

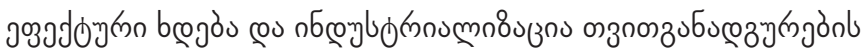

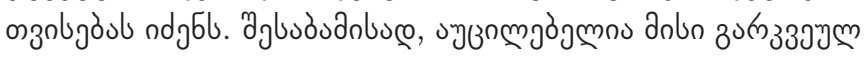

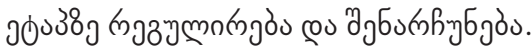

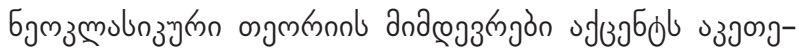

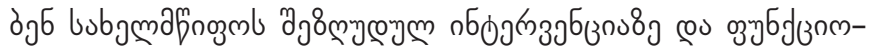

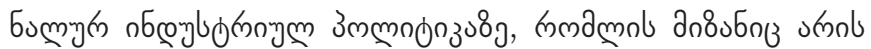

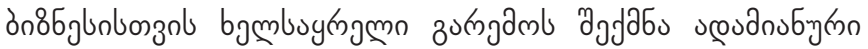

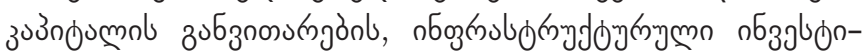

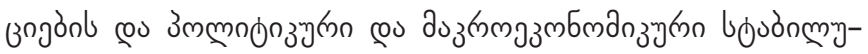

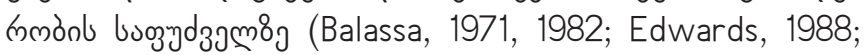
Wolf, 1988; (0) ง.D).

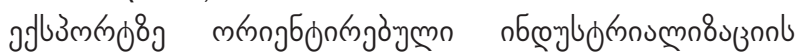

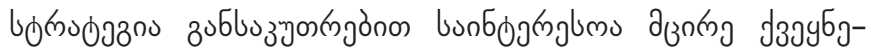

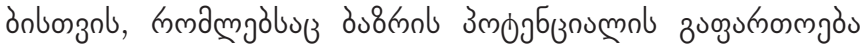

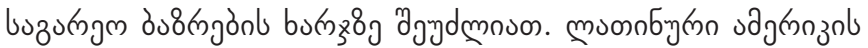

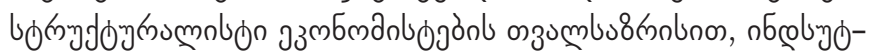

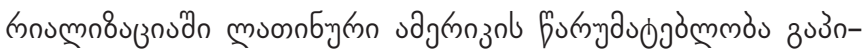

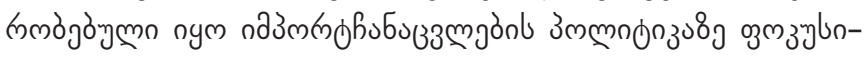

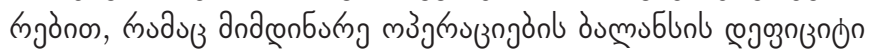

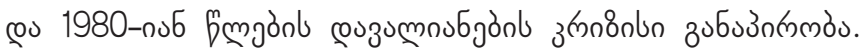

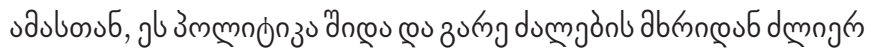

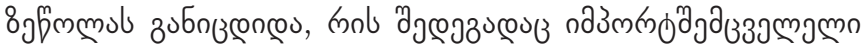

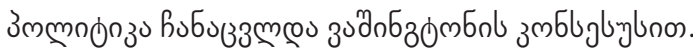

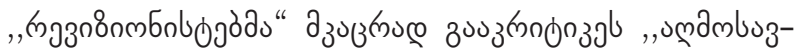

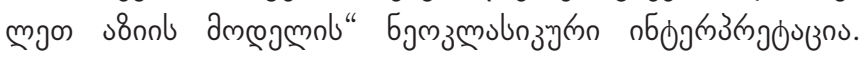
aszumnonse, s. salı zubnbnmuzl zm

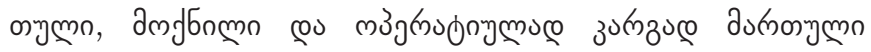

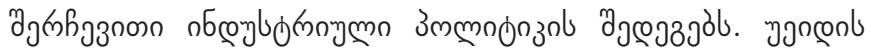

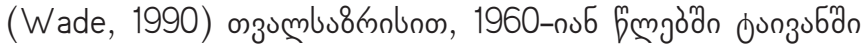

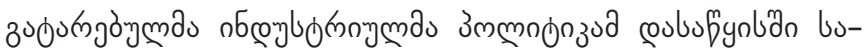




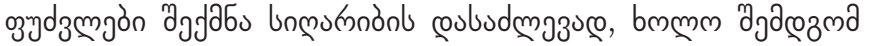

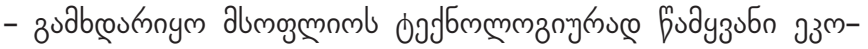

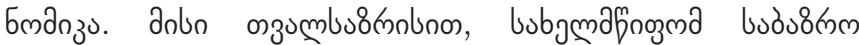

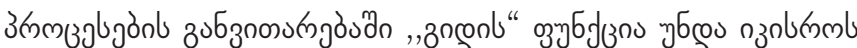

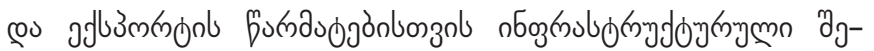

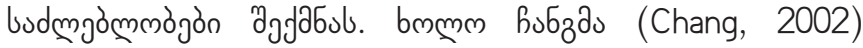

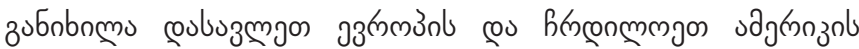

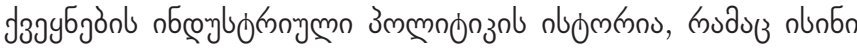

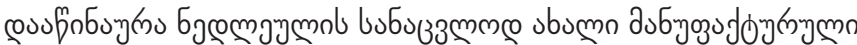

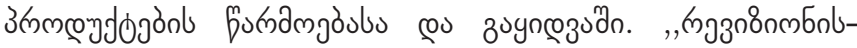

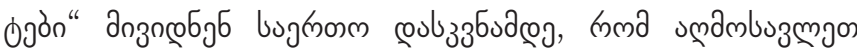

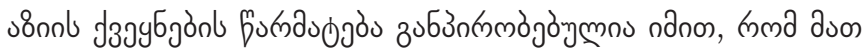

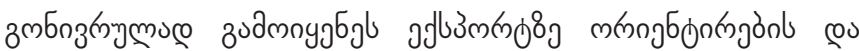

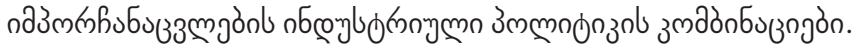

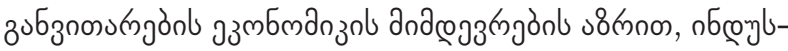

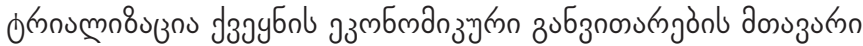

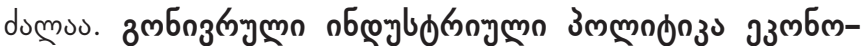

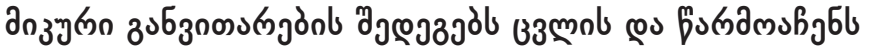

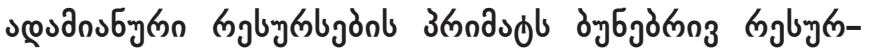

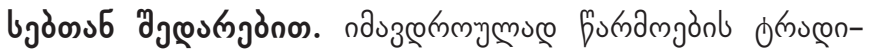

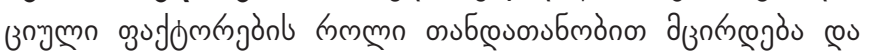

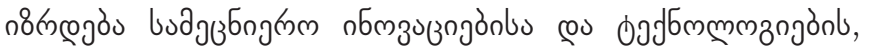

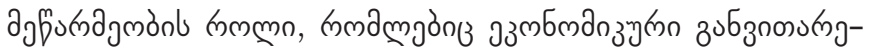

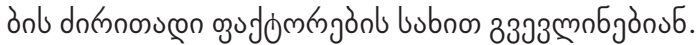

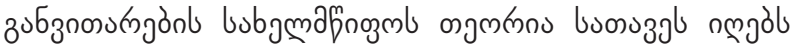

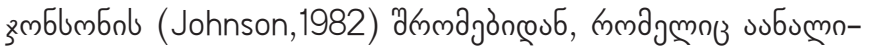

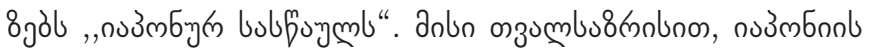

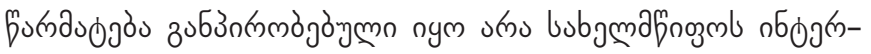

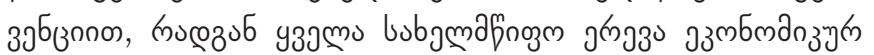

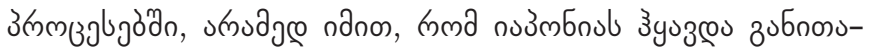

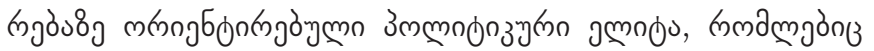

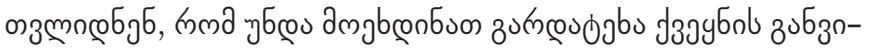

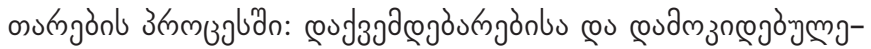

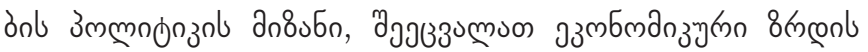

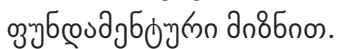

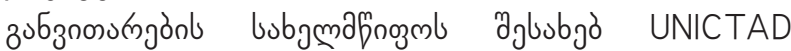

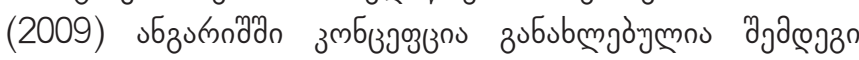

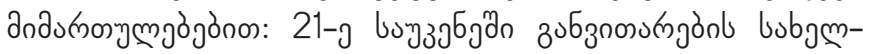

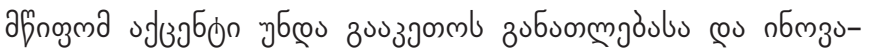

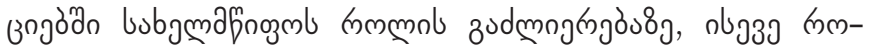
zмm

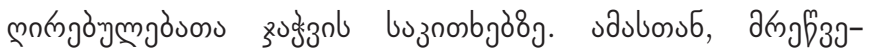

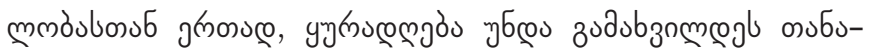

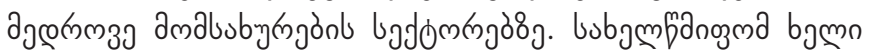

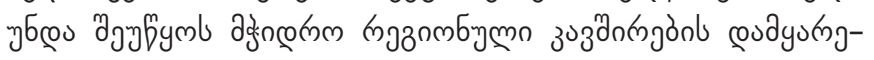

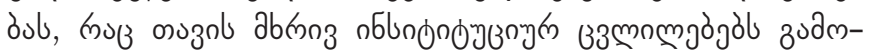

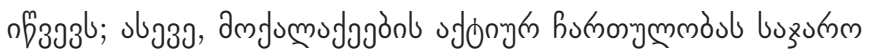

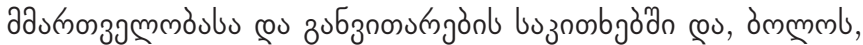

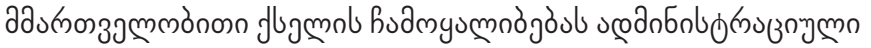

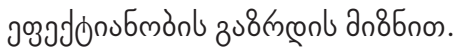

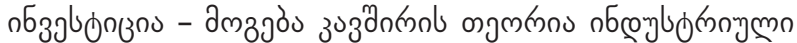

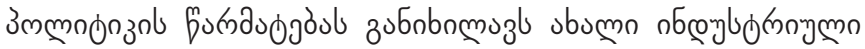

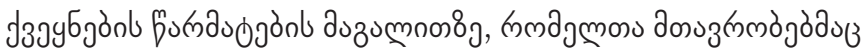

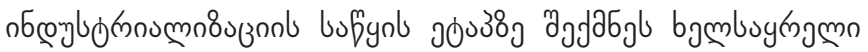

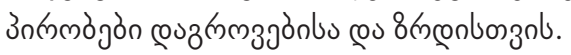

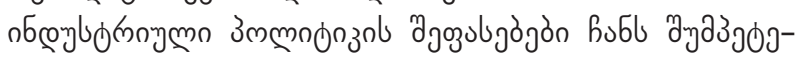

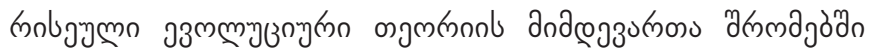

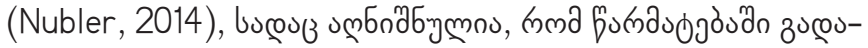

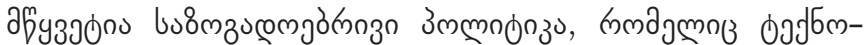

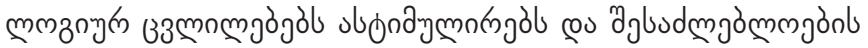

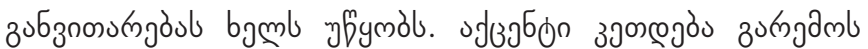

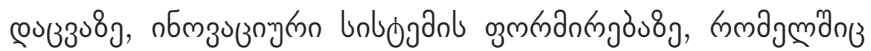

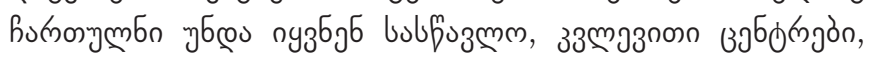

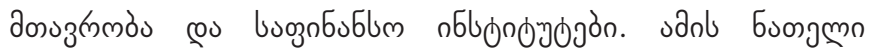

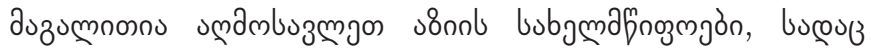

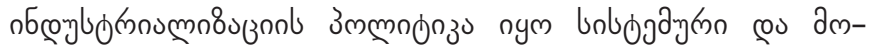

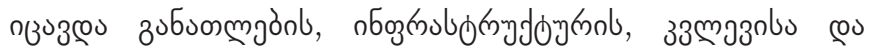

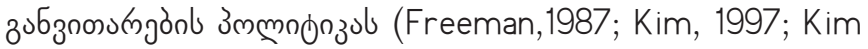
\& Nelson, 2000, Lee, 2015). ason s8

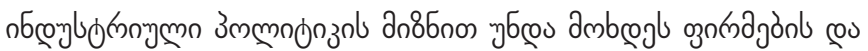

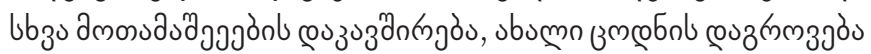

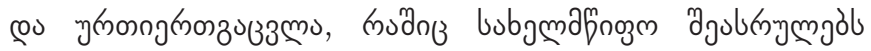

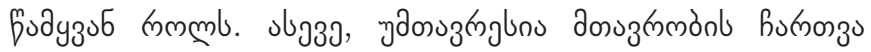

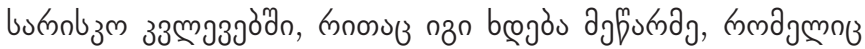

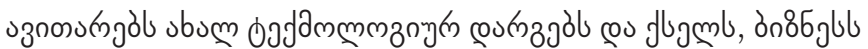

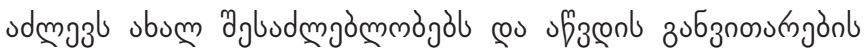
zindjamzunons bjozul.

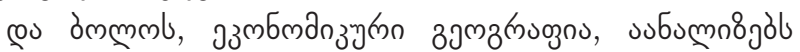

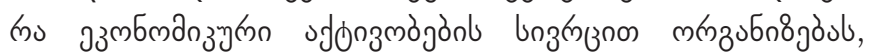

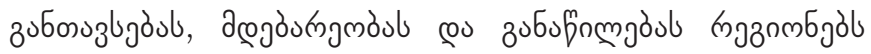

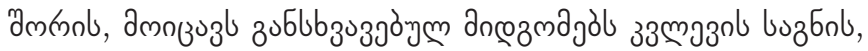

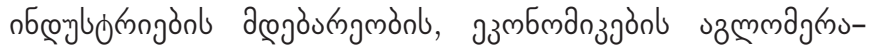

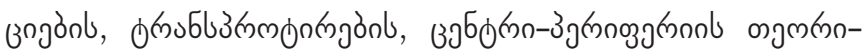

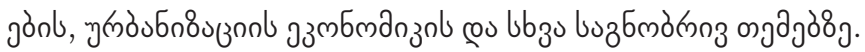

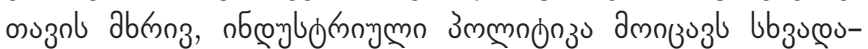

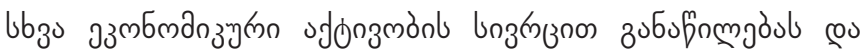

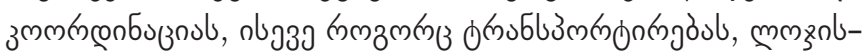

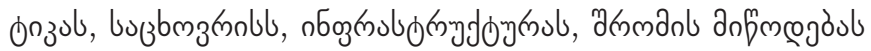

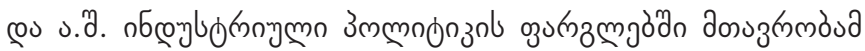

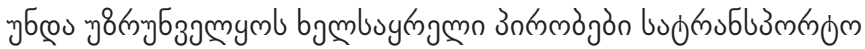

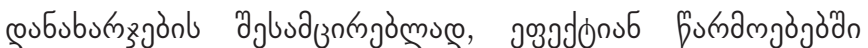

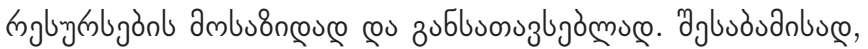

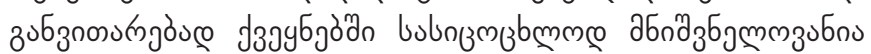

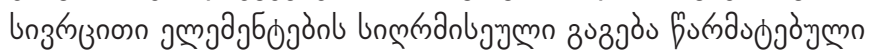

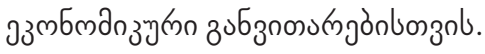

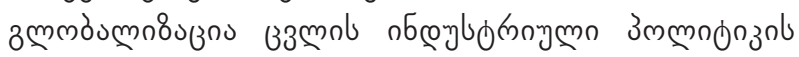

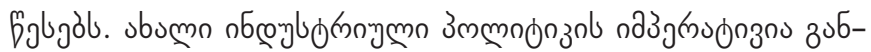

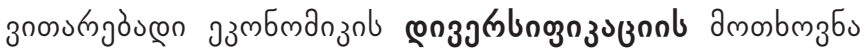

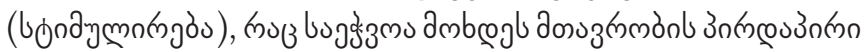

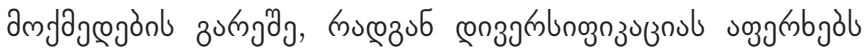




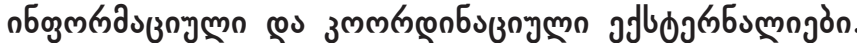

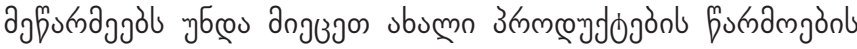

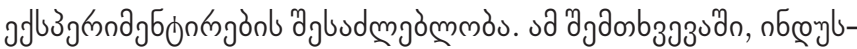

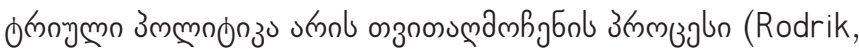

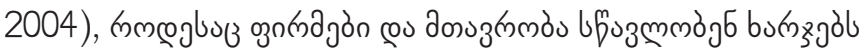

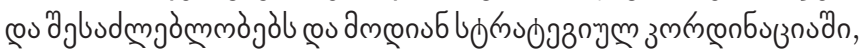

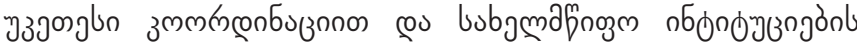

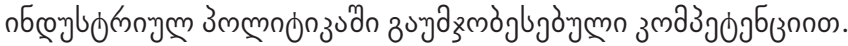

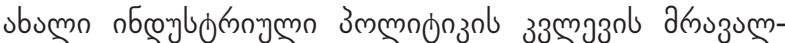

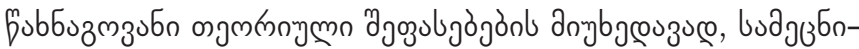

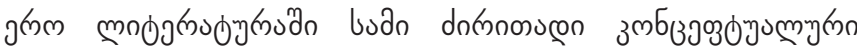

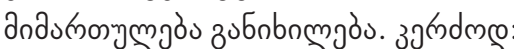

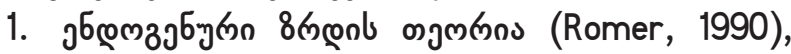

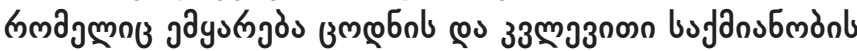

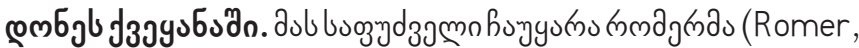

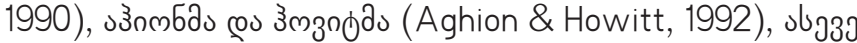

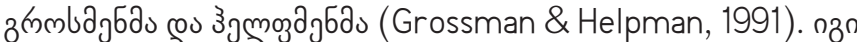

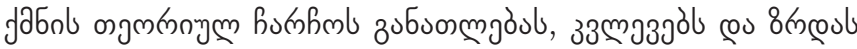

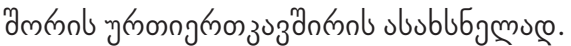

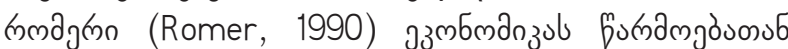

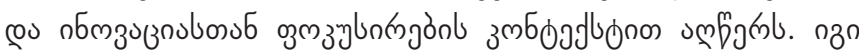

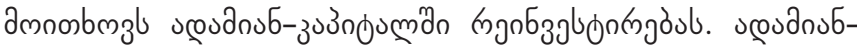
зuउnoumann nб

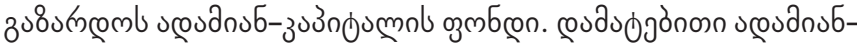

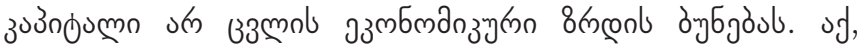

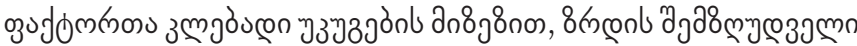
dumgàn anjagejòjб.

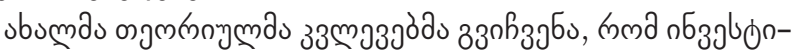

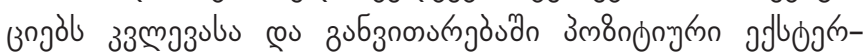

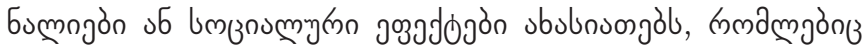
oुn

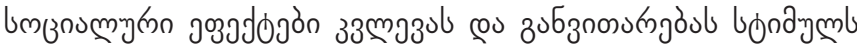

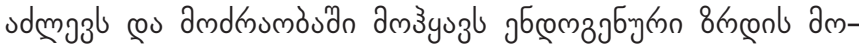

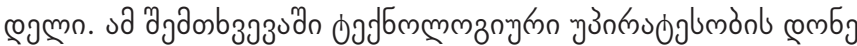

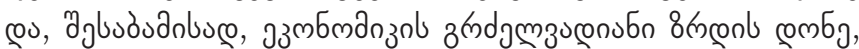

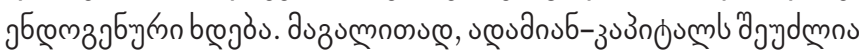

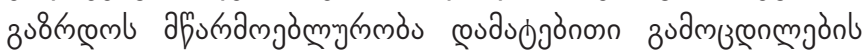

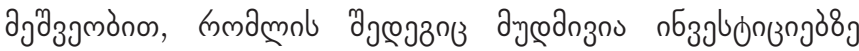

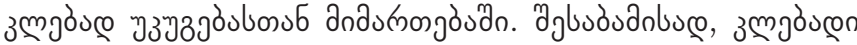

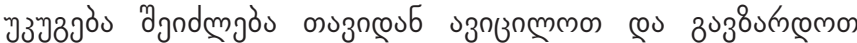

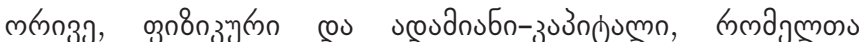

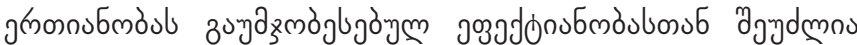

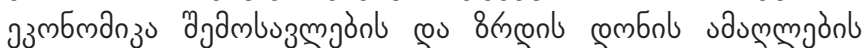

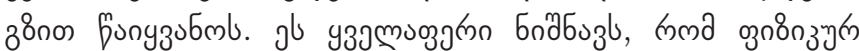

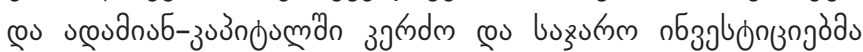

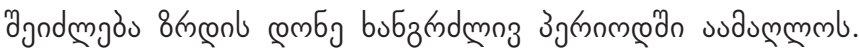

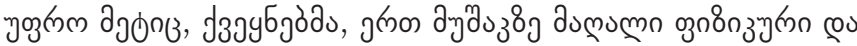

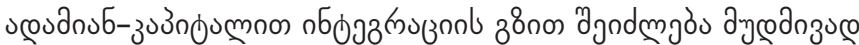

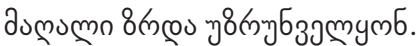

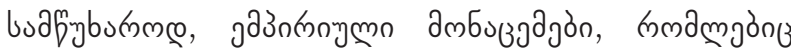

चз山3

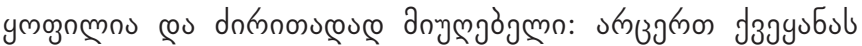

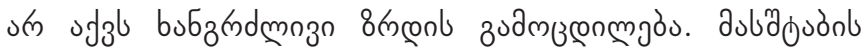

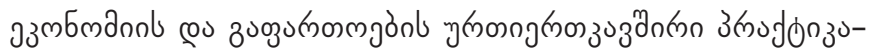

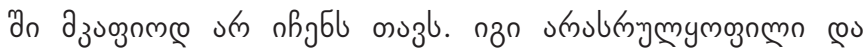

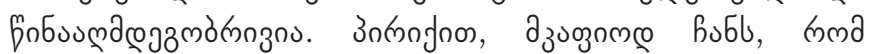

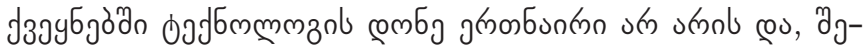

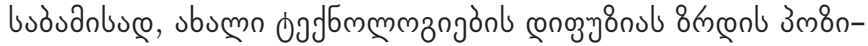

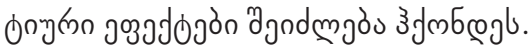

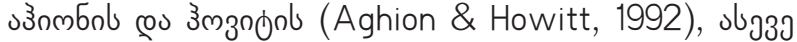

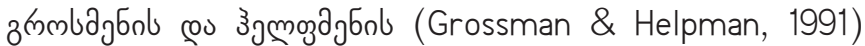

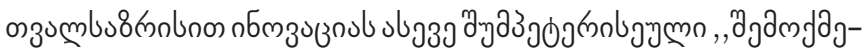

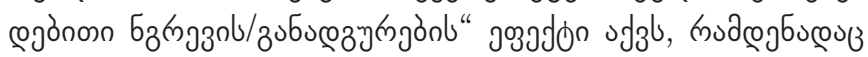

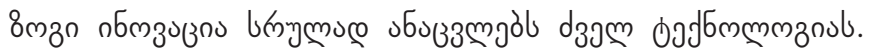

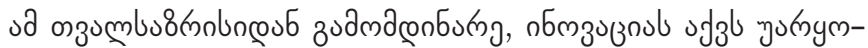

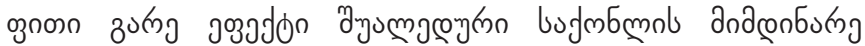

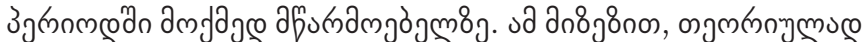

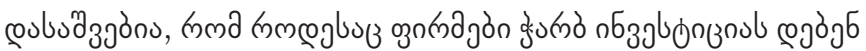

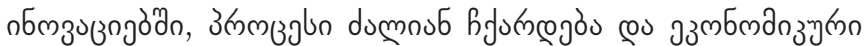

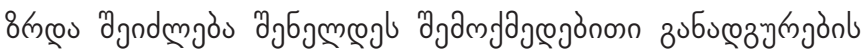
उऊmmighn zodm.

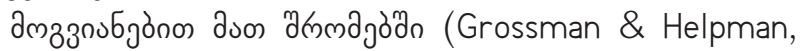

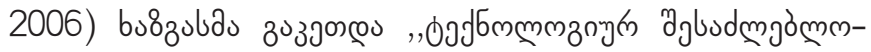

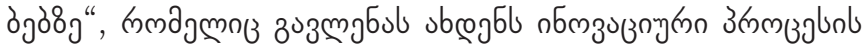

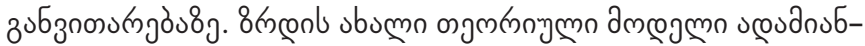

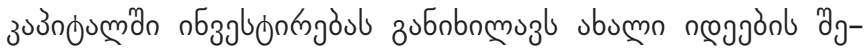

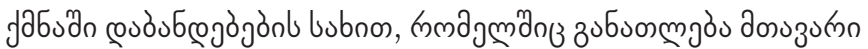

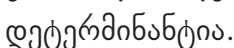

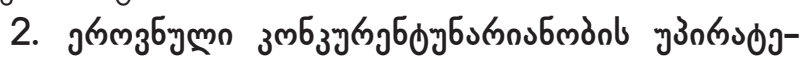

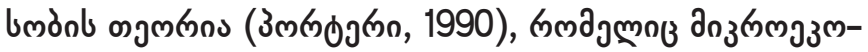

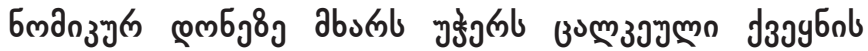

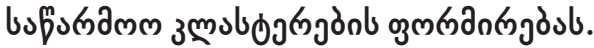

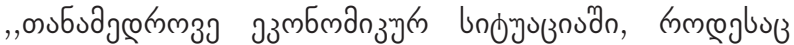

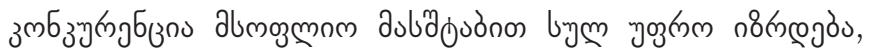

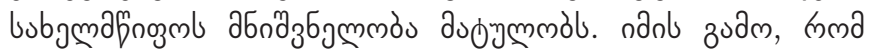

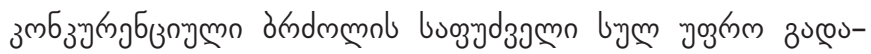

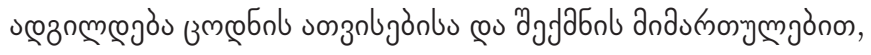

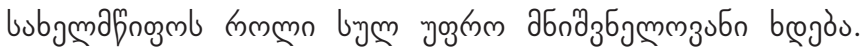

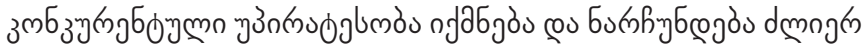

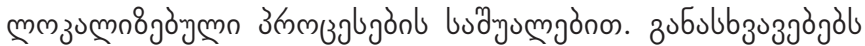

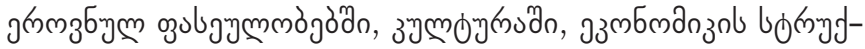

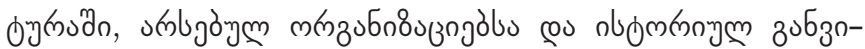

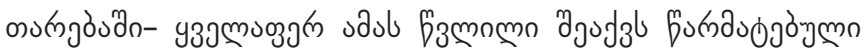

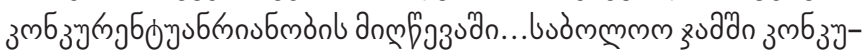

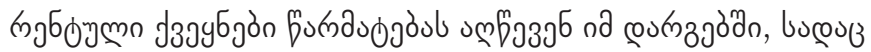

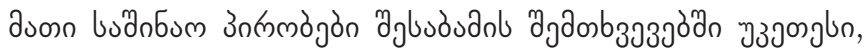

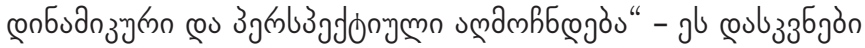

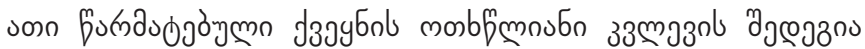
(Porter, 2004; 162).

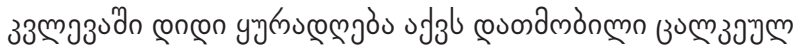




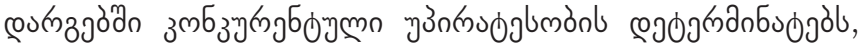

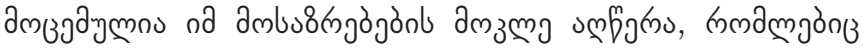

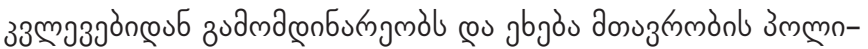

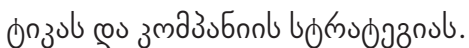

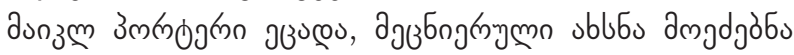

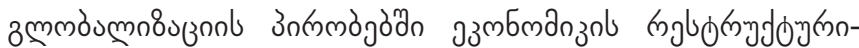

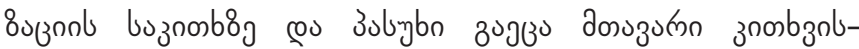

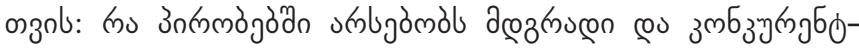

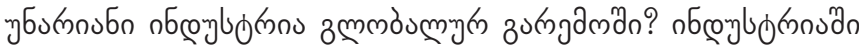

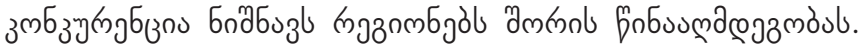

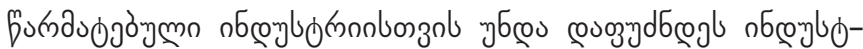

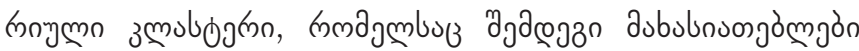

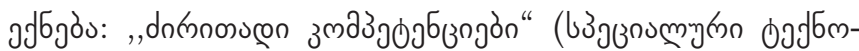

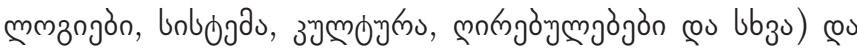

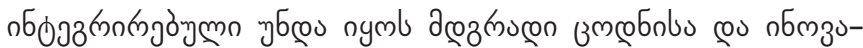

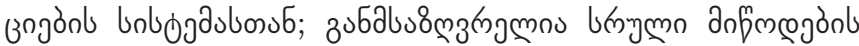

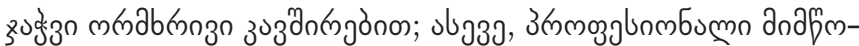

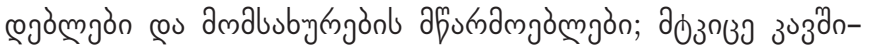

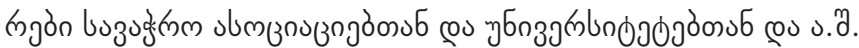

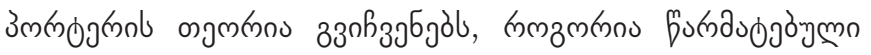

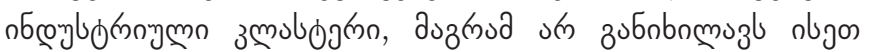

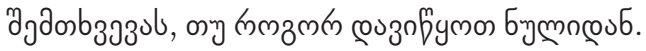

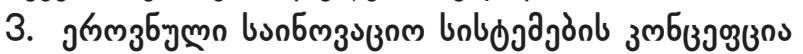

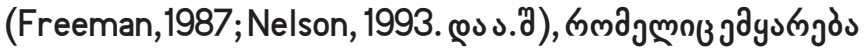

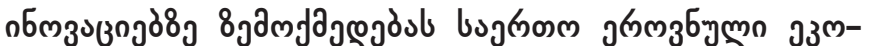

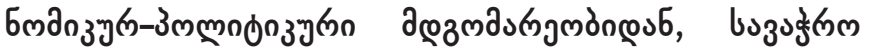

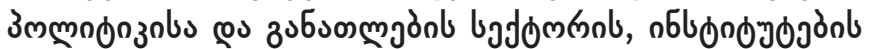

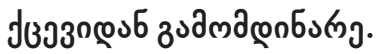

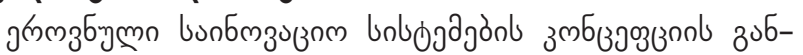

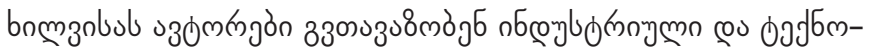

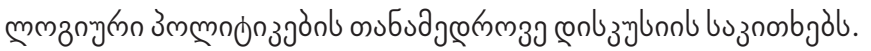
dumnoर abnдз

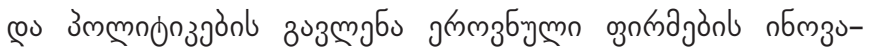

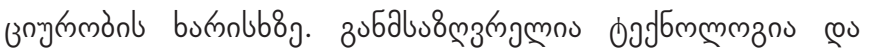

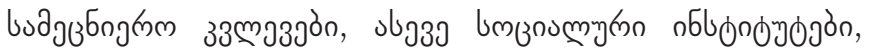

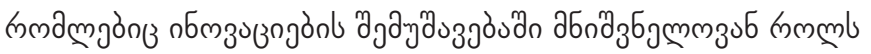

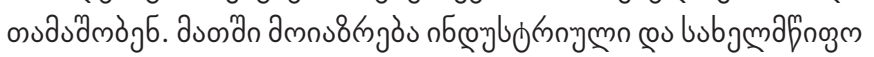

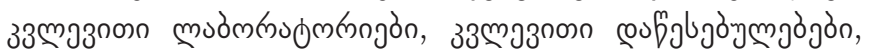

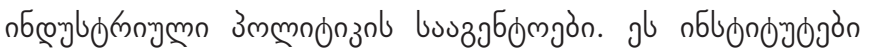

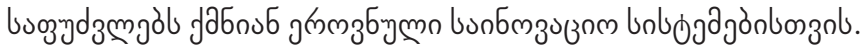

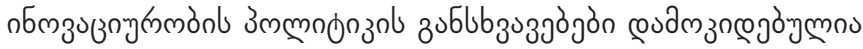

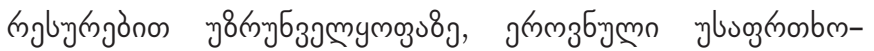

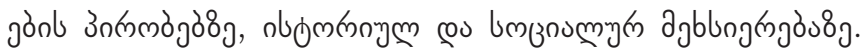

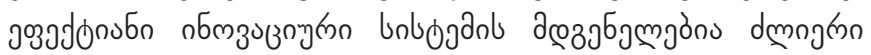

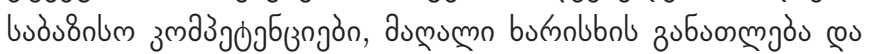
o cou buzuğkn उ̈mmndozu.

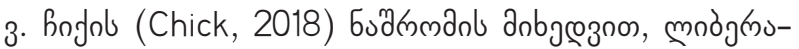

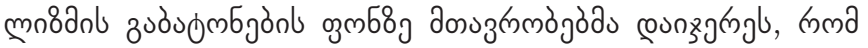

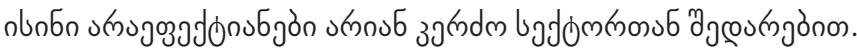

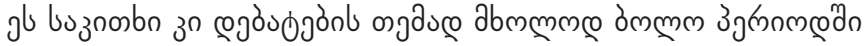

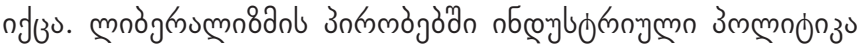

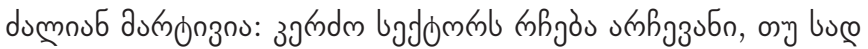

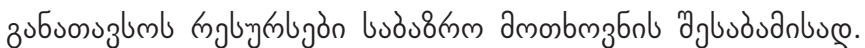

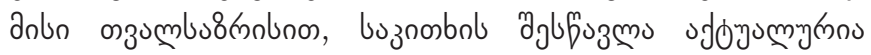

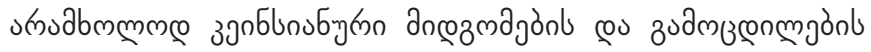

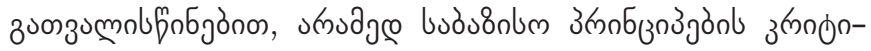
उचmmn zubbnm

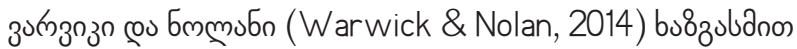

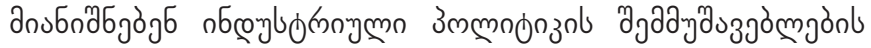

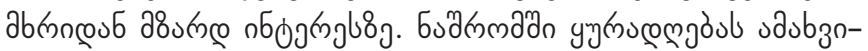

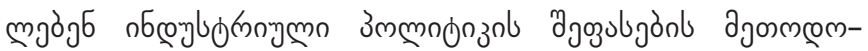

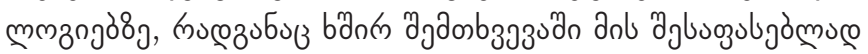

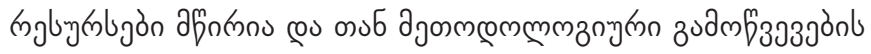

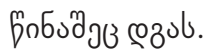

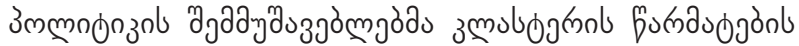

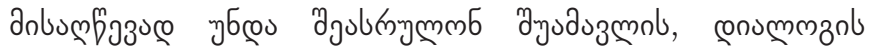

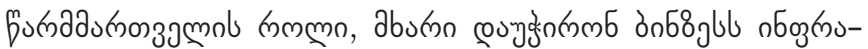

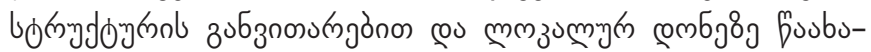

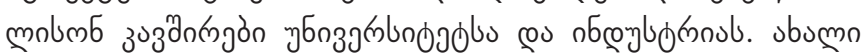

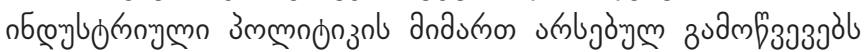

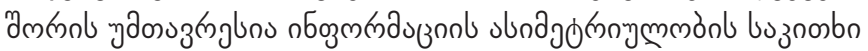

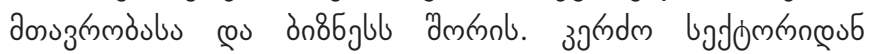

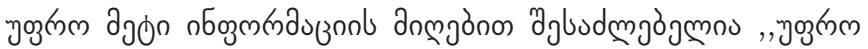

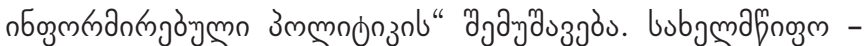

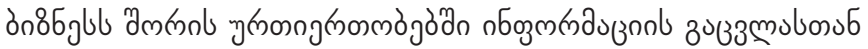
j grosuce du mou

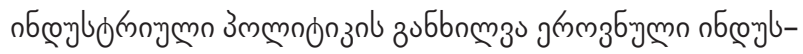

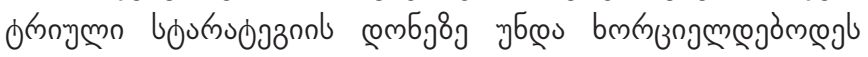

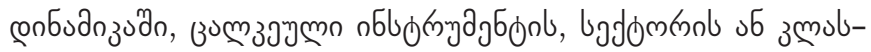

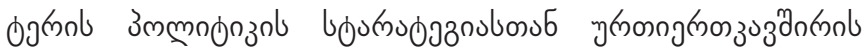

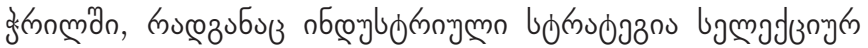

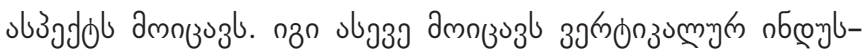

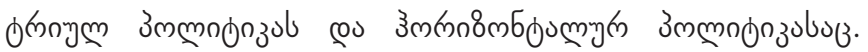

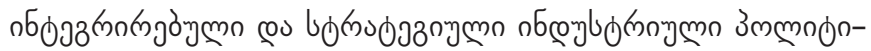

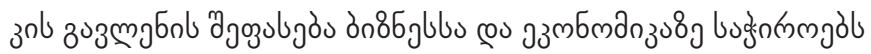

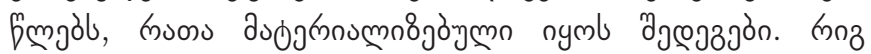

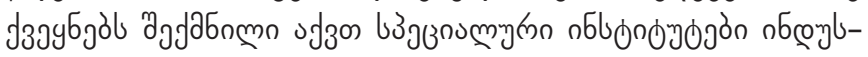

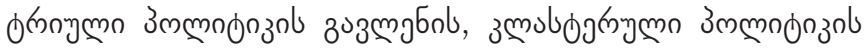

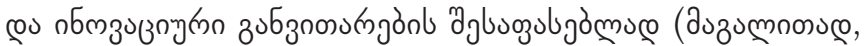

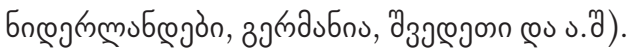

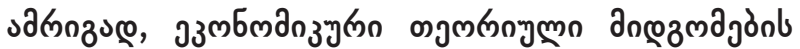

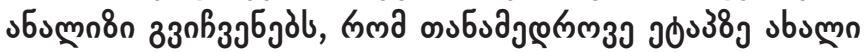

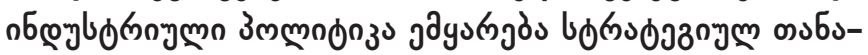

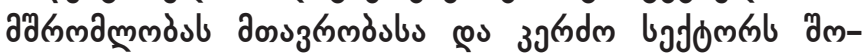

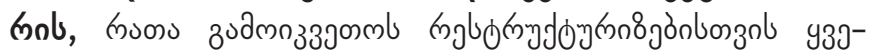

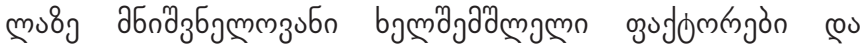

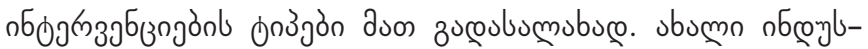

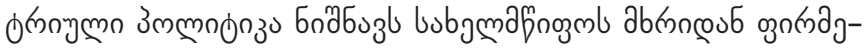

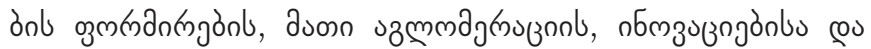




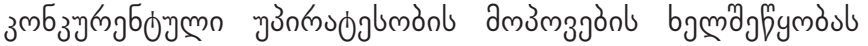

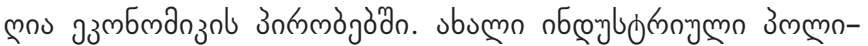

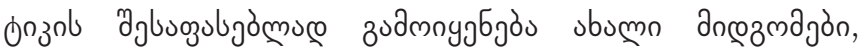

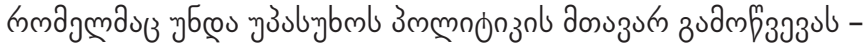

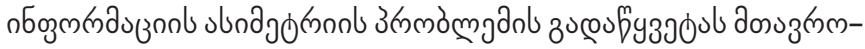

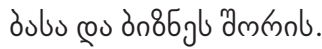

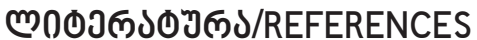

Lekashvili, E. (2018). For the Actualization of Scientific Study on New Industrial Policy, Third International Scientifical Conference, Challenges of Globalization in Economics and Business", TSU Faculty of Economics and Business, 25-26/ Oct./. Conference Proceedings. pp. 237-240 (In Georgian).

Aghion, P. and Howitt, P. A Model of Growth Through Creative Destruction. Econometrica, Vol. 60, No. 2 (Mar., 1992), pp. 323-351 Published by: The Econometric Society Stable URL: http://www.jstor.org/stable/2951599;

Amsden, A. (1989). Asia's Next Giant: South Korea and Late Industrialization. Oxford University Press. New York and Oxford, UK. Balassa, B. (1971). The Structure of Protection in Developing Countries. Johns Hopkins University Press. Baltimore.

Gagnidze, I. (2016) The Impact of Entrepreneurial Universities on the Innovative Development of Economy, $\neg$ III International scientific and practical conference "Strategic Imperatives of Modern Management", KNEY, Kiev, 2016, pp. 186-192. http://wiki.kneu.kiev.ua/bitstream/2010/20956/1/186-192.pdf

Chang, HJ. (2009). Industrial policy: Can we go beyond an unproductive confrontation? Paper presented at the Annual World Bank . Conference on Development Economics, 22-24 June, Seoul, Republic of Korea. Available at: http://siteresources. worldbank.org/INTABCDESK2009/Resources/Ha-Joon-Chang.pdf.

Chick Victoria, Industrial Policy, then and now, https://rwer.wordpress.com/comments-on-rwer-issue-no-84/ (18.12.18).

Edwards, S.(1988). Exchange Rate Misalignment in Developing Countries. John Hopkins University Press. Baltimore.

Freeman, C. (1987). Technology, Policy, and Economic Performance: Lessons from Japan. Pinter Publishers. London.

Grossman, G. and Helpman, E. (1991). Innovation and Growth in the Global Economy. MIT Press Cambridge, MA.

Johnson, C (1982). MITI and the Japanese Miracle: The Growth of Industrial Policy, 1925-1975. Stanford University Press. Stanford, CA.

Kim, L.(1997). Imitation to Innovation: The Dynamics of Korea's Technological Learning. Harvard Business School Press. Boston.

Kim, L, and Nelson, RR. (2000). Technology, Learning and Innovation: Experiences of Newly Industrializing Economies. Cambridge University Press. Cambridge, UK.

Lee, K. (2015). Industrial policies for upper-middle-income countries. E15Initiative. International Centre for Trade and Sustainable Development (ICTSD) and World Economic Forum. Geneva. Available at:http://e15initiative.org/publications/ industrial-policiesfor-upper-middle-income-countries/

Lin, JF. (2011). From flying geese to leading dragons: New opportunities and strategies for structural transformation in developing countries. Policy Research Working Paper No. 5702. World Bank. Washington, DC.

Lin, JF, and Chang HJ (2009). Should industrial policy in developing countries conform to comparative advantage or defy it? A debate between Justin Lin and Ha-Joon Chang. Development Policy Review 27(5): 483-502.

Lin JY., and Treichel, V. (2014). Making industrial policy work for development.

Porter, M.(2004)., Competitive Strategy, technices for analysing industries and copmpetitors, The Free Press.

Porter, M. (1990). The Competitive Adventage of Nation, The Free Press;

Nelson, R. (1993). National Innovation Systems: A Comparative Analysis. University of Illinois at Urbana-Champaign ss Academy for Entrepreneurial Leadership Historical Research Reference in Entrepreneurship. Available at SSRN: https:// ssrn.com/abstract=1496195

Nubler, I. (2014). A theory of capabilities for productive transformation: Learning to catch up

Pack, H., and Saggi ,K. (2006). Is there a case for industrial policy? A critical survey. The World Bank Research Observer 21(2): 267-97.

Rodrik, D. (2004). Industrial policy for the twenty-first century. CEPR Discussion Paper 4767. Centre for Economic Policy Research. London.

Romer, M. Paul. (1990). Endogenous Technological Change. Journal of Political Economy, Vol. 98, No. 5, Part 2: The Problem of Development: A Conference of the Institute for the Study of Free Enterprise Systems (Oct., 1990), pp. S71-S102 Published by: The University of Chicago Press Stable URL: https://www.jstor.org/stable/2937632

Should Industrial Policy in Developing Countries Conform to Comparative Advantage or Defy it? A Debate Between Justin Lin and Ha-Joon Chang (2009) 
UNCTAD (2009). Least Developed Countries Report 2009: The State and Development Governance. United Nations. Geneva and New York.

Wade, R. (1990). Governing the Market. Economic Theory and the Role of Government in East Asian Industrialization. Princeton University Press. Princeton, NJ.

Wade, R. (2010). After the Crisis: Industrial Policy and the Developmental State in Low-Income Countries, London School of Economics and Political Science. Global Policy. pp.150-156.

Warwick, K. (2013). Beyond industrial policy: Emerging issues and new trends. OECD Science, Technology and Industry Policy Paper No. 2. Organization for Economic Cooperation and Development. Paris.

Warwick, K. et A. Nolan (2014), "Evaluation of Industrial Policy: Methodological Issues and Policy Lessons ", OECD Science, Technology and Industry Policy Papers, No. 16, Éditions OCDE, Paris. DOI: http://dx.doi.org/10.1787/5jz181jh0j5k-en Wolf, C .(1988). Markets or Governments: Choosing between Imperfect Alternatives. MIT Press. Cambridge, MA. 